\title{
Observations sur des cas de myiase canine à Cordylobia anthropophaga Blanchard à Bobo-Dioulasso, République de Haute-Volta
}

\author{
par R. GIDEL, E. LE BERRE ef A.' CHALLIER
}

\begin{abstract}
RÉSUMÉ
Un cas de myiase à Cordylabia anthropophaga chez un chien adulte et trois cas chez des chıots sont décrits.

Après avoir étudié la symptomatologie et indiqué la thérapeutique mise en œuvre, les auteurs relatent le processus de contamination et son importance épidémiologique pour l'homme nolamment.

La durée du cycle larvaire dans les cas rapportés est d'environ un mois. Des essais d'élevage à partir de larves transplantées sur rats blancs ef de larves extraites à leur maturité ont abouti à la constitution d'une colonie adulte qui n'a pu avoir de descendance.
\end{abstract}

\section{INTRODUCTION}

A la fin de la saison sèche à Bobo-Dioulasso, il a été observé quatre cas de myiase canine à Cordylobia anthropophaga Blanchard. Les chiens parasités ( 3 chiots d'une même portée et un chien adulte) appartenaient à deux familles européennes résıdant dans le même quartier.

Les larves de Cordylobio anthropophaga, appelées communément «vers de Cayor», sont trouvées assez fréquemment chez l'homme, certains animaux domestiques (chien, chat, chèvre, lapin, cobaye) et des animaux sauvages (petits mammifères surtout), de différentes parties d'Afrique au Sud du Sahara (ZUMPT, 1965).

\section{2. ÉTUDE DES CAS OBSERVÉS : SYMPTOMATOLOGIE ET TRAITEMENT}

1 er cas : Chien adulte.

Ce cas fut observé chez un chien mâle, âgé de 12 ans, croisé de griffon et de fox anglais.

Le 8 mars l'animal présenta des signes d'as- thénie avec une démarche vacillante et une difficulté à se déplacer. L'appétit était cependant conservé. A partir du 12 mars, le chien commença à manifester de l'agitation : il ne restait pas en place ef recherchait la présence de lieux humides: flaques d'eau de pluie, bac à douche... Les signes d'asthénie s'accentuèrent en même temps que l'animal perdait son appétıt et maigrissalt rapidement. Les seuls sympiômes observés à l'examen clinique étaient une anémie intense et une tachycardie. Un examen coprologique ne montra rien d'anormal, tandıs qu'un hémogramme effectué le 14 mars se présentait comme suit :
Hémoglobine

Nombre d'hématıes

Nombre de leucocytes ...........

Polynucléares neutrophiles......

Polynucléaires éosinophıles .....

Polynucléaires basophiles .......

Lymphocytes. . . . . . . . . . . . . .

Monocyies

sur 100 éléments comptés.
45 p. 100

2.200 .000

7.300 
A la suite de cet examen, le traitement suivant fut institué : Hépatrol injectable, 1 ampoule par jour; Vitamine B12 à 1.000 gammas, 1 ampoule par jour; Quotivit O.E., 1 comprimé par jour : Vitascorbol, 1/2 comprimé par jour.

En dépit de ce traitement, aucun signe d'amélioration ne fut observé. Au contraire, l'état de l'anımal continua à empirer ef il cessa complètement de s'alimenter. Toutefois, il accepta de boire l'eau sucrée qui lui était présentée.

Le 19 mars, tout en poursuivant le traitement précédent, une thérapeutique hormonale fut mise en œuvre. L'animal reçut une injection de testostérone (25 mg) - folliculine (lmg), suivie de trois autres injections à 48 heures d'intervalle. Le 22 mars, les premiers signes d'amélioration furent notés. L'animal, quı était complètement amorphe, commença à s'animer en présentant des signes de satisfaction à l'arrivée de ses maîtres (battements de queve, grognements), tandis que son appétit revenait progressivement. Lorsque l'animal parvint à se déplacer, il rechercha à nouveau les lieux humides.

Début avril, l'état du chien s'était nettement amélioré. II avait retrouvé son appétit et reprenait du poids. Le traitement fut alors modifié ainsi : Hépatrol-Sorbitol, 1 ampoule tous les 2 jours; Vitamine B12 à 100 gammas, 1 ampoule par jour: Quotivit O.E., 1 comprimé tous les 2 jours; Vitascorbol, $1 / 2$ comprimé tous les 2 jours.

Le 13 avril, l'animal semblant rétabli, le traitement d'entretien suivant fut alors préconisé : Hépatrol buvabie, Chophytol gouttes, Survitine et Vitascorboi.

Le 16 avril, de petits nodules sous-cutanés apparurent au niveau des oreilles. A la pression, il sortit de chacun d'eux une larve de mouche. Celles-ci furent identifiées comme étant des larves de Cordylobia anthropophaga au deuxième stade. Le 17 avril, I'animal se mit à boiter de la patte antérieure gauche qui était œématiée au niveau des coussinets plantaires et des espaces interdigıtaux. Des larves de deuxième stade furent extraites en grand nombre de cette région. Du 18 au 22 avril, de nombreux nodules apparurent successivement sur le corps, mass plus particulièrement sous le museau, au coin de l'œil gauche, au niveau des lèvres ainsi que sur le flanc gauche et les deux pattes antérieures. Par contre, une seule larve fut rencontrée sur la queve et 3 ou 4 seviement sur les pattes postérieures. Aucune larve ne fut trouvée sur les testicules. Notons que dans sa position de repos, l'animal se couchait sur le flanc gauche.

Un deuxième hémogramme fuł effectué le 20 avrıl, c'est-à-dire au moment de la sortie des larves. Il se présentait ainsi :

Hémoglobıne...............

Nombre d'hématies .......... 3.000.000

Nombre de leucocytes ......... $\quad 20.300$

Polynucléaires neutrophiles...... 88

Polynucléares éosınophıles ..... 0

Polynucléaires basophiles .......

Lymphocytes. . . . . . . . . . . . . . $\quad 10$

Monocytes .................. 2

pour 100 éléments comptés.

Bien que l'animal n'ait à aucun moment reçu d'antibiotiques, sa température resta constamment normale.

Un troisième hémogramme, effectué 5 semaines plus tard (1 er juin), montra que tout était rentré dans l'ordre :

Hémoglobine.

60 p. 100

Nombre d'hématies ........... 4.040 .000

Nombre de leucocytes ......... $\quad 6.800$

Polynucléaires neutrophiles...... 74

Polynucléaires éosinophiles ..... 3

Polynucléaires basophiles .......

Lymphocytes.................. 18

Monocytes ................. 5

pour 100 éléments comptés.

\section{$2^{\mathrm{e}}$ cas : Chiots.}

Ce cas fut observé chez 3 chiots d'une même portée, âgés de 1 mois. Un seul d'entre eux nous fuł présenté pour furonculose, les deux autres animaux présentant les mêmes sıgnes. L'animal était couvert de nodules de la taille d'un petit pois renfermant une ou piusieurs larves de Cordylobio anthropophaga au troisième stade. Ces nodules se rencontraient sur le flane droit, sur la paupière inférieure drolte, les lères, le museau, l'oreille droite, et les pattes antérieures et postérieures droites. Plus de 70 larves furent recueillies chez cet animal; après leur extraction, les plaies furent désınfectées soigneusement, celles-cl étant en effet plus importantes que dans le premier cas, car il s'agissait de larves du $3^{\text {e }}$ stade et de surcroît beaucoup de nodules en 
renfermaient plusieurs. Le traitement suivant fut mis en cuvre pour les 3 chiots : sirop de Cyclomycétine, Ultralevure, Vitascorbol, Vitamine B12, Survitine.

Malgré l'intensité de ce parasitisme, les trois chiots se rétablirent rapıdement, sans complications.

Notons qu'aucune larve ne fut trouvée chez la mère.

\section{OBSERVATIONS SUR LES LARVES ET LES ADULTES DE CORDYLOBIA ANTHROPOPHAGA}

Les larves ef les adultes que nous avons examinés correspondaient aux descriptions de ZUMPT (1965).

Nous avons essayé d'élever cette espèce d̀ partir de larves récoltées sur le chien adulte et sur le chiot.

MACDONALD (1962) a transplanté des larves de second stade et de début du $3^{\mathrm{e}}$ stade chez des rats blancs. Nous avons utilısé sa technique très simple qui consiste, à l'aide d'une sonde, à pratiquer des cavités entre la peau ef les muscles. Nous avons transplanté ainsi sous la peau de l'abdomen de 4 rats blancs une quinzaine de larves. Parmi ces dernières, se trouvaient quelques spécimens au stade III ; leurs péritrènes éfaient encore visibles lorsqu'elles se sont logées dans la cavité; les larves plus petites se sont enfouies plus profondément sous la peau.

Une dizaine de jours après le transplant, 5 larves sont tombées pour puper, dans les plateaux disposés sous les cages des rats. Ces pupes provenaient des iarves les plus grosses. Comme après trols semaines d'expérience les autres larves ne tombaient pas, nous avons examiné les rats. La majorité des cavités pratiquées s'était refermée sur les petites larves profondément enfoncées. Ces dernières se sont enkystées; les kystes avaient la forme d'une sphère très dure de 5 à $7 \mathrm{~mm}$ de diamètre. A la dissection, nous avons trouvé un magma blanchâtre résultant de la lyse des tissus larvaires et le tégument chiffoné que l'on reconnaît à ses épines norres.

Les adultes ont été placés dans une cage, au laboratoire. La température de ce local, climatisé une partie de la journée, variait de $22^{\circ} \mathrm{C}$ à $30^{\circ} \mathrm{C}$ environ. La noturriture était du jus d'orange. Un plateau de sable sec a été placé dans la cage pour que les femelles y déposent leurs œufs. Aucune descendance n'a pu être obtenue de ce premier essai.

Un second lot de larves provenant du jeune chiot a été mıs en élevage. Des 70 larves extraites le même jour de la peau de l'hôte, 50 exemplaires de $3^{\mathrm{e}}$ stade ont été déposés sur le sable sec d'un plateau; en quelques minutes toutes les larves se sont enfoncées pour puper, sauf deux qui sont demeurées à la surface et sont mortes. Il semble que ces deux larves et les huit qui se sont enfoncées, mals n'ont pas pupé, avaient été lésées lors de leur extraction de la peau. 39 adultes ont éclos, tous en même temps, 13 jours après l'extraction des larves. II y avalt autant de femelles que de mâles. Nous les avons nourris avec le jus d'orange et nous avons observé des accouplements. Le plateau rempli de sable et destiné à la ponte avait été préalablement placé sous des cobayes pour que leur urine imprègne le sable. ZUMPT (1965) signale en effet que le sable souillé par l'urine et les fèces incite les femelles à pondre. Tous les jours, nous avons examıné le sable à la loupe bınocularre et le plateau a été placé plusieurs fois sous des cobayes; aucun animal n'a été infesté. La colonie s'est éteinte 12 jours après l'éclosion.

Dans son ouvrage intıtulé «Myısis in man and animals in the old world » (1965), ZUMPT décrit toute la biologie de l'espèce déjà étudiée par BLACKLOCK et THOMPSON (1923). II signale qu'à la température du laboratore, les adultes éclosent après $10-11$ jours ( 13 jours pour nos résultats) et que lo femelle vit environ deux semaines et rarement jusqu'à trois.

\section{4. ÉTUDE DU PROCESSUS DE CONTAMINATION}

\section{1. Observations antérieures.}

Les femelles déposent leurs œufs en des lieux ombragés sur du sable sec ou des vêtements malpropres; jamais les œufs ne sont déposés directement sur la peau (ZUMPT, 1965). Les larves éclosent après 3 jours et peuvent survivre 9 et parfois 15 jours sans prendre de nourriture. Lorsqu'un objet touche la surface du sable, les larves sortent rapidement. S'il s'agit d'un hôte, 
elles se fixent à la peau et la perforent. La durée de la pénétration peut varier de 25 secondes à une demi-heure. La larve demeure ensuite au point de pénétration en position sous-cutanée. Elle se nourrit aux dépens des tissus de l'hôte et subit deux mues. Chez le rat, la durée des stades larvaires est d'environ 9 jours.

\section{2. Observations sur le chien adulte.}

Le chien adulte parasité avait été placé dans une niche neuve contenant une litière de sable sec, le 5 mars. Les premiers signes de fatigue sont apparus le 8 mars et les larves ont été abservées le 16 avrıl. Le chien couchait auparavant dans l'appartement carrelé de ses maîtres. Il est fondé de penser que les premıers signes de fatigue correspondent à la phase d'invasion des larves et que la contamination a eu lieu très peu de temps après l'occupation de la niche. La durée du développement larvarre du stade œuf au 3e stade larvaire a donc été de 5 à 6 semaines.

\section{3. Observations sur les chiots.}

La mère des trois chiols parasités était indemne et avait mis bas un mois avant l'extraction des larves sous un hangar dont le sol étalt sablonneux. Comme cette chienne vivait habituellement dans l'appartement de ses mâtres, elle y a ramené ses trols chıots dès le deuxième jour. La date présumée de contamination doit donc se situer immédıatement après la naissance.

\section{4. Contamination de l'homme.}

On connaît depuis longtemps le «ver de Cayor » (BLANCHARD, 1893). Récemment LECLERCQ (1966) a constaté deux cas de cette myiase chez des enfants belges revenant du
Congo. Or bien souvent, les enfants disposent de bassins remplis de sable et situés dans un coin ombragé du jardin. Ce milieu présente des conditions très favorables pour la ponte de l'insecte.

\section{CONCLUSION}

Les observations que nous avons exposées nous amènent d̀ considérer quatre points :

1. La durée des cycles larvaires dans les deux cas décrits est comprise entre 30 et 40 jours. Chez le chien cette durée serait donc plus longue que chez le rat et le cobaye ( 8 à 9 jours).

2. La croissance larvare peut engendrer chez l'hôte, dans le cas d'un parasitisme intense, des troubles graves, et notamment une anémie prononcee.

3. Cette myiase furonculeuse peut ne pas être rapportée à sa véritable cause et, de ce falt, l'insecte bouclera son cycle et sera à l'origine de nouveaux foyers.

4. Le «ver de Cayor » bien connu des populations autochtones d'Afrique Occidentale semble être ignoré dans le milieu européen. II seralt souhaltable que ce dernier soit informé du danger que peut représenter la présence d'aires sablonneuses dans les jardins, niches ou autres lieux.

Organisation de Coopération

et de Coordination pour la lutte contre les Grandes Endémies (O.C.C.G.E.)

Centre Muraz, Bobo-Dioulasso,

Rép. de Houte-Volto.

Mission de l'Office de la Recherche Scientifique et Technique Outre-Mer (O.R.S. T. O.M.) auprès de I'O.C. C. G.E.

\title{
SUMMARY
}

Observations on some cases of myiasis due to Cordylobia anthropophoga Blanchard, in dog, in Bobo-Dioulasso, Republic of Upper Volta

\begin{abstract}
A case of myiasis due to Cordylobia anthropophago in an adult dog and 3 cases in puppies have been described.

The symptoms of this infestation, the treatment, which has been carried out. the transmission and its consequence on the epidemiology of the disease in man, have been reported. In the cases reported, the larval cycle was lasting about a month. Rearing trials, made with larvae transplanted on white rat and larvae taken out when mature, resulted in the establishment of an adult population which could not have any lineage.
\end{abstract}




\section{RESUMEN}

Observaciones sobre casos de miasıs canına con Cordylobia anthropophaga Blanchard en Bobo-Dioulasso, República de Alta-Yolta

Se describen un caso de miasis con Cordylobia onhropophaga en un perro adulto y tres casos en cachorros.

Se estudia la sintomatologia y se indıca la terapéutica utilizada. Después, los autores notan el proceso de conlaminación y su importancıa epidemiologica, particularmente para el hombre.

Es cosa de un mes la duración del ciclo larvario en los dichos casos. Ensayos de cria a partir de larvas trasplaniadas sobre ratas blancas y de larvas extraidas al momento de su madurez acertaron. Pero la colonia adulta obtenida no pudo tener descendencia.

\section{BIBLIOGRAPHIE}

BLACKLOCK (B.), THOMPSON (M. G.). - A study on the tumbu fly, Cordylobia anthropophaga Grünberg, in Sierra Leone. Ann. trop. Med. Porosit., 1923, 17, 443-502.

BLANCHARD (R.). - Contribution à l'étude des diptères parasites et sur une larve de coléoptère vomie par un enfant, au Sénégal. Bull. Soc, ent. Fr., 1893, 1.

LECLERCQ $\left(M_{1}\right)$. - Myiase cutanée furoncu- leuse par Cordylobia anthropophaga Blanchard constatée en Belgique. Rev. Méd. de Liège, 1966, 21, 12-13.

MCDONALD (W. A.). - A technique for rearing myiasis-producing fly larvae. J. ent. Soc. S. Afr. 1962, 25, 149.

ZUMPT (F.). - Myasis in man and animals in the old World. London, Butterworth, 1965, 267 pages. 